\title{
Central Nervous System Hodgkin Lymphoma
}

National Cancer Institute

\section{Source}

National Cancer Institute. Central Nervous System Hodgkin Lymphoma. NCI Thesaurus.

Code C114951.

A Hodgkin lymphoma that arises from the central nervous system. 